\title{
Multi parametric model predictive control based on laguerre model for permanent magnet linear synchronous motors
}

\author{
Nguyen Hong Quang ${ }^{1}$, Nguyen Phung ${ }^{2}$, Dao Phuong $\mathrm{Na}^{3}$, Nguyen Thanh Binh ${ }^{4}$ \\ ${ }^{1}$ Departement of Automation, Thai Nguyen University of Technology, Viet Nam \\ ${ }^{2}$ Institude for Control Engineering and Automation, Hanoi University of Science and Technology, Viet Nam \\ ${ }^{3}$ Departement of Automatic Control, Hanoi University of Science and Technology, Viet Nam \\ ${ }^{4}$ University of Ulsan, South Korea
}

\begin{tabular}{l} 
Article Info \\
\hline Article history: \\
Received May 262018 \\
Revised Sep 13, 2018 \\
Accepted Oct 10, 2018 \\
\hline Keywords: \\
Model predictive control \\
Multi parametric programming \\
Permanent magnet linear \\
synchronous motor \\
Polysolenoid linear motor
\end{tabular}

Corresponding Author:

Nguyen Hong Quang,

Department of Automation,

Thai Nguyen University of Technology,

666, 3/2 Street, Tich Luong ward, Thai Nguyen city, Viet Nam.

Email: quang.nguyenhong@tnut.edu.vn

\begin{abstract}
The permanent magnet linear motors are widely used in various industrial applications due to its advantages in comparisons with rotary motors such as mechanical durability and directly creating linear motions without gears or belts. The main difficulties of its control design are that the control performances include the tracking of position and velocity as well as guarantee limitations of the voltage control and its variation. In this work, a cascade control strategy including an inner and an outer loop is applied to synchronous linear motor. Particularly, an offline MPC controller based on MPP method and Laguerre model was proposed for inner loop and the outer controller was designed with the aid of nonlinear damping method. The numerical simulation was implemented to validate performance of the proposed controller under voltage input constraints.
\end{abstract}

Copyright $@ 2019$ Institute of Advanced Engineering and Science. All rights reserved.

\section{INTRODUCTION}

The Permanent Magnet Linear Synchronous Motors (PMLSM) is extensively used in various industries due to the ability to directly create linear motion without gears or belts. Although the mediate mechanical actuators are eliminated, the system become weak robustness, in that external impact such as frictional force, end - effect, changed load and non-sine of flux cause damage to control performances. Generally, principle operation of synchronous linear motor is similar to permanent magnet synchronous motor; however their physical construction is different in [1]-[5], and various applications such as CNC Lathe [6], sliding door [7].

In recent years, there has been many researches for control problem of permanent magnet linear motor. An adaptive fuzzy neural network in [8] was proposed to control the permanent magnet linear synchronous motor. The authors in [9] presented a control design to regulate velocity based on PI - self tuning combining with appropriate estimation technique at slow velocity zone, but if load is changed, PI self tuning controller will be not efficient. In order to overcome changed load, model reference control method based on Lyapunov stability theory employed in [10]. Additionally, the backstepping technique in [11], was applied to reduce influence of frictional force and controller is designed based on appropriate frictional estimated model. In [12], the advantage of that the sliding mode control applied in Linear Motor is that real position value tracks set point. However, the disadvantages of this method is that sliding surface is complicated and chattering problem occurred. It is clear that the previous researches do not mention position, velocity and current constraints. To solve this problem, the MPC approach in [13] was proposed as a single 
controller for speed control. The authors in [14] built a new mathematic model and use optimal control approach to result in linear quadratic regulation (LQR). However, the considered model did not include disturbance load as well as friction force. In addition, the implementation of this MPC controller on a microcontroller is very difficult because of calculation burden.

In this paper, we apply cascade control strategy to synchronous linear motor including an inner and an outer loop. The offline MPC controller based on MPP method in [15] was proposed for inner loop to make motor current to follow the reference signal from the outer controller. We modify optimization problem in the MPC controller by using a Laguerre Model approach in [13] to reduce the number of optimal variables. The major advantage of our MPC controller lies in the ability to solve constraints problem and reducing amount of calculation because the optimal problem is offline solved. The outer controller was designed based on nonlinear damping method in [16] to guarantee the error between real and reference velocity converge to arbitrary small value.

\section{PRIMARY RESULTS}

\subsection{Laguerre orthogonal polynomials}

As represented in [13] Laguerre polynomials are defined as follows:

$$
l_{i}(z)=\sqrt{\left(1-a^{2}\right)} \frac{\left(z^{-1}-a\right)^{i-1}}{\left(1-a z^{-1}\right)^{i}}
$$

where $a$ is a positive constant, $0 \leq a<1$ and $i=0,1, \ldots$. The application of Laguerre polynomials is mainly in the area of system identification, in which the discrete-time impulse response of a dynamic system is represented by a Laguerre model (see Wahlberg, 1991). In this work, based on Wang 2009, we obtain the main result: Suppose that the impulse response of a stable system is $H(k)$, then with a given number of terms $N, H(k)$ is written as:

$$
H(k)=c_{1} l_{1}(k)+c_{2} l_{2}(k)+\ldots+c_{N} l_{N}(k)
$$

with $c_{1}, c_{2}, \ldots, c_{N}$ are the coefficients to be determined from the system data. The discrete-time Laguerre functions are orthonormal functions, and with these orthonormal properties, the coefficients of the Laguerre network are defined by the following relation:

$$
c_{i}=\sum_{k=0}^{\infty} H(k) l_{i}(k), \forall i \in\{0,1, \ldots, N\}
$$

\subsection{Model predictive control based on Laguerre function}

In this section, we consider this discrete time linear system described by:

$$
x_{m}(k+1)=A_{m} x_{m}(k)+B_{m} u(k)
$$

In which, $x_{m}$ is vector of state variable and $u(k)$ is the input at the time $k$.

Convert the Equation (1) as follows:

$$
\Delta x_{m}(k+1)=A_{m} \Delta x_{m}(k)+B_{m} \Delta u(k)
$$

Where: $\Delta x_{m}(k+1)=x_{m}(k+1)-x_{m}(k), \Delta u(k)=u(k)-u(k-1)$

From (4), (5) and by letting $x(k+1)=\left[\Delta x_{m}(k+1) x_{m}(k+1)\right]^{T}$, the Equation (5) becomes:

$$
x(k+1)=A x(k)+B \Delta u(k)
$$


With: $A=\left[\begin{array}{cc}A_{m} & \Theta_{n \times n} \\ A_{m} & I_{n \times n}\end{array}\right], \quad B=\left[\begin{array}{l}B_{m} \\ B_{m}\end{array}\right]$

Predictive model of (6) at $k_{i}$ as follows:

$$
\left\{\begin{array}{l}
x(k+i+1 \mid k)=A x(k+i \mid k)+B \Delta u(k+i \mid k) \\
i=0,1, \ldots, N \\
x(k \mid k)=x(k)
\end{array}\right.
$$

$N$ is prediction horizon. From (7), the sake of designing the MPC controller is finding the sequence of input signal $u(k)$ minimizing this under cost function:

$$
J=\sum_{j=1}^{N_{p}} x(k+j \mid k)^{T} Q x(k+j \mid k)+\sum_{j=0}^{N_{p}} \Delta u(k+j)^{T} R \Delta u(k+j)
$$

Where $Q, R$ are positive definite matries.

Denote that:

$$
\begin{aligned}
X & =\left[\begin{array}{llll}
x(k+1 \mid k)^{T} & x(k+2 \mid k)^{T} & \cdots & x(k+N \mid k)^{T}
\end{array}\right]^{T} \\
U & =\left[\begin{array}{llll}
\Delta u(k \mid k)^{T} & \Delta u(k+1 \mid k)^{T} & \cdots & \Delta u(k+N-1 \mid k)^{T}
\end{array}\right]^{T}
\end{aligned}
$$

Putting (7) into a form admitting variable (9) as;

$$
X=\hat{A} x_{0}+\hat{B} U
$$

Where:

$$
\hat{A}=\left[\begin{array}{c}
A \\
A^{2} \\
A^{3} \\
\vdots \\
A^{N}
\end{array}\right], \hat{B}=\left[\begin{array}{ccccc}
B & 0 & 0 & 0 & 0 \\
A B & B & 0 & 0 & 0 \\
A^{2} B & A B & B & 0 & 0 \\
\vdots & \vdots & \vdots & \ddots & 0 \\
A^{N-1} B & A^{N-2} B & \cdots & A B & B
\end{array}\right]
$$

and $x_{0}=x(k)$ is initial state of prediction horizon. Substituting (10) into the cost function (8):

$$
J=\left(\frac{1}{2} U^{T} H U+x_{0}^{T} F U+x_{0}^{T} \hat{A}^{T} \hat{Q} \hat{A} x_{0}\right)
$$

Where:

$$
H=2\left(\hat{B}^{T} \hat{Q} \hat{B}+\hat{R}\right), F=2 \hat{A}^{T} \hat{Q} B^{T}, \hat{Q}=\left[\begin{array}{cccc}
Q & \Theta & \Theta & \Theta \\
\Theta & Q & \Theta & \Theta \\
\vdots & \vdots & \ddots & \Theta \\
\Theta & \Theta & \Theta & P
\end{array}\right], \hat{R}=\left[\begin{array}{cccc}
R & \Theta & \Theta & \Theta \\
\Theta & R & \Theta & \Theta \\
\vdots & \vdots & \ddots & \Theta \\
\Theta & \Theta & \Theta & R
\end{array}\right]
$$

In this work, we regard the sequence of signals $\Delta u(k \mid k), \Delta u(k+1 \mid k), \ldots, \Delta u(k+N-1 \mid k)$ can be approximated by the by a discrete Laguerre polynomial functions. In other words, a set of Laguerre functions $l_{1}(k), l_{2}(k), \ldots, l_{M}(k)$ are used to capture the input $\Delta u_{i}(k+i \mid k)$ in prediction horizon: 


$$
\Delta u_{i}(k+i \mid k)=\sum_{j=1}^{M} c_{j} l_{j}(i)
$$

Where $c_{j}$ are the coefficients which depend only on the initial of prediction horizon. $l_{j}(i)$ are the orthogonal Laguerre functions having form as following:

$$
\eta=\left[\begin{array}{cc}
c_{1}^{1}(k) & c_{2}^{1}(k) \ldots . c_{M}^{1}(k) \\
c_{1}^{2}(k) & c_{2}^{2}(k) \ldots . . c_{M}^{2}(k) \\
\vdots \\
c_{1}^{n}(k) & c_{2}^{n}(k) \ldots . . c_{M}^{n}(k)
\end{array}\right]^{T}, L=\left[\begin{array}{cccc}
l_{1}(0) & l_{2}(0) & \ldots & l_{M}(0) . \\
l_{1}(1) & l_{2}(1) & \ldots & l_{M}(1) . \\
& & & \\
l_{1}(N-1) & l_{2}(N-1) & \ldots & l_{M}(N-1)
\end{array}\right]^{T}
$$

The equation (12) is rewrited to:

$$
\Delta u\left(k_{i}+k\right)=L(k)^{T} \eta
$$

Substuting (13) model (4), we can obtain:

$$
x\left(k_{i}+j \mid k_{i}\right)=A^{j} x\left(k_{i}\right)+\sum_{i=0}^{j-1} A^{j-i-1} B \Delta u(k+i)=A^{j} x(k)+\sum_{i=0}^{j-1} A^{j-i-1} B L(i)^{T} \eta=A^{j} x(k)+\phi(j)^{T} \eta
$$

Where $\phi(j)^{T}=\sum_{i=0}^{j-1} A^{j-i-1} B L(i)^{T}$

Substituting into the cost function we obtain:

$$
\begin{aligned}
J & =\sum_{j=1}^{N_{p}}\left(A^{j} x\left(k_{i}\right)+\phi(j)^{T} \eta\right)^{T} Q\left(A^{j} x\left(k_{i}\right)+\phi(j)^{T} \eta\right)+\sum_{j=0}^{N_{p}}\left(L\left(k_{i}+j\right)^{T} \eta\right)^{T} R\left(L\left(k_{i}+j\right)^{T} \eta\right) \\
& =\eta^{T}\left(\sum_{j=1}^{N_{p}} \phi(j) Q \phi(j)^{T}+\sum_{j=0}^{N_{p}} L\left(k_{i}+j\right) R L\left(k_{i}+j\right)^{T}\right) \eta+2 \eta\left(\sum_{j=1}^{N_{p}} \phi(j) Q A^{j}\right) x\left(k_{i}\right)+\sum_{j=1}^{N_{p}} x\left(k_{i}\right)^{T}\left(A^{T}\right)^{m} Q A^{m} x\left(k_{i}\right)
\end{aligned}
$$

Let: $\Omega=\sum_{j=1}^{N_{p}} \phi(j) Q \phi(j)^{T}+\sum_{j=0}^{N_{p}} L(j) R L(j)^{T} ; \psi=\sum_{j=1}^{N_{p}} \phi(j) Q A^{j}$

We set the optimization problem as:

$$
\eta^{*}=\arg \min \left(\eta^{T} \Omega \eta+2 \eta^{T} \psi x\left(k_{i}\right)\right)
$$

\section{- Unconstrained MPC controller}

In this case, (7) was considered without any constraints on inputs and state variables:

$$
\frac{\partial J}{\partial \eta}=2 \eta^{T} \Omega+2 \psi x\left(k_{i}\right)
$$

Which is equivalent:

$$
\eta^{*}=-\Omega^{-1} \psi x\left(k_{i}\right)=-\left(\Omega=\sum_{j=1}^{N_{p}} \phi(j) Q \phi(j)^{T}+\sum_{j=0}^{N_{p}} L(j) R L(j)^{T}\right)^{-1} \sum_{j=1}^{N_{p}} \phi(j) Q A^{j} x\left(k_{i}\right)
$$


So that, the optimal input signal at time $k_{i}$ :

$$
\Delta u\left(k_{i}\right)=L(0)^{T} \eta^{*}
$$

- Constrained MPC controller

We suppose that (14) has constraints as follows:

$$
\begin{aligned}
& u_{\min } \leq u(k) \leq u_{\max } \\
& x_{m \text { min }} \leq x_{m}(k) \leq x_{m \text { max }}
\end{aligned}
$$

We consider prediction model (7) and constraints (15) at time $k_{i}$ :

$$
\begin{aligned}
& u_{\text {min }}-u\left(k_{i}-1\right) \leq \Delta u\left(k_{i} \mid k_{i}\right) \leq u_{\max }-u\left(k_{i}-1\right) \\
& {\left[\begin{array}{c}
x_{m \min }-x_{m}\left(k_{i}\right) \\
x_{m \min }
\end{array}\right] \leq x\left(k_{i}+1 \mid k_{i}\right) \leq\left[\begin{array}{c}
x_{m \max }-x_{m}\left(k_{i}\right) \\
x_{m \max }
\end{array}\right]}
\end{aligned}
$$

(15), (16) deduce to:

$$
\Delta U_{\min } \leq \Delta u\left(k_{i}\right) \leq \Delta U_{\max }
$$

Furthermore, by using $\Delta u\left(k_{i}\right)=L(0)^{T} \eta$, rewriting (17):

$$
M \eta \leq \gamma
$$

Thus, the optimization problem (14):

$$
\begin{aligned}
& \min J=\min \eta^{T} \Omega \eta+2 \eta^{T} \psi x\left(k_{i}\right) \\
& \text { s.t. } M \eta \leq \gamma
\end{aligned}
$$

Since $J$ is the quadratic function, optimal issue in (19) can be solved by Quadratic Programming (QP) to obtain the solution $\eta^{*}$, the control signal is calculated by:

$$
\Delta u\left(k_{i}\right)=L(0)^{T} \eta^{*}
$$

\subsection{Multi parametric programming}

In this section, we remind the results of multi parametric programming method (MPP) in [11]. The basic idea is that the space of parameter is separated into critical regions in that each critical regions, the solution of optimization problem is in the same form. Consider the quadratic cost function:

$$
\begin{aligned}
& V^{*}=\min _{z}\left(\frac{1}{2} z^{T} H z\right) \\
& \text { s.t. } G z \leq W+S \theta
\end{aligned}
$$

with $z \in \square^{s}$ is the vector of optimization variables. $\theta \in \square^{n}$ is the vector of parameters, and the matrices: $H \in \square^{s \times s}, G \in \square^{q \times s}, W \in \square^{q}, S \in \square^{q \times n}$. Let $D$ be a polytopic set of parameters. In multi parametric programming, we consider finding the solution of optimizing $V$ on $D$. By using first order Karush-KuhnTucker (KKT) optimality conditions we obtain:

$$
\begin{aligned}
& H z+G^{T} \lambda=0, \lambda \in \square^{q} \\
& \lambda_{i}\left(G^{i} z-W^{i}-S^{i} \theta\right)=0, i=1,2, \ldots, q
\end{aligned}
$$




$$
\lambda \geq 0
$$

where $i$ is the row index. From (22) we have:

$$
z=-H^{-1} G^{T} \lambda
$$

Substituting into (23) we arrive at the conditions as follow:

$$
\lambda_{i}\left(-G^{i} H^{-1} G^{i T} \lambda_{i}-W^{i}-S^{i} \theta\right)=0, i=1,2, \ldots, q
$$

Let $\breve{\lambda}$ and $\tilde{\lambda}$ are the Lagrange multiples corresponding to the inactive constraints and active constraints respectively. With the inactive constraints we have $\breve{\lambda}=0$. With the active constraints we receive:

$$
\tilde{\lambda}=-\left(\tilde{G} H^{-1} \tilde{G}^{T}\right)^{-1}(\tilde{W}+\tilde{S} \theta)
$$

Where $\tilde{G}, \tilde{W}, \tilde{S}$ are the matrices corresponding to the active constraints. There exist $\left(\breve{G} H^{-1} \tilde{G}^{T}\right)^{-1}$ because the rows of $\tilde{G}$ is linear independence. Substituting $\tilde{\lambda}$ from (24) into (21) we obtain:

$$
z^{*}(x)=H^{-1} \tilde{G}^{T}\left(\tilde{G} H^{-1} \tilde{G}^{T}\right)^{-1}(\tilde{W}+\tilde{S} \theta)
$$

And from (20), $\tilde{\lambda}$ in (24) must be satisfied:

$$
\tilde{\lambda}=-\left(\tilde{G} H^{-1} \tilde{G}^{T}\right)^{-1}(\tilde{W}+\tilde{S} \theta) \geq 0
$$

The results (25) and (26) are the basic of multi parametric programming method in this case. Based on the above results, the main steps of the off-line mp-QP solver are outlined in the following algorithm [11]:

Step 1: Defining the current region be the whole space $D$ of the vector of parameter $\theta$.

Step 2: Choose vector $\theta_{0}$ in the current region.

Step 3: With $\theta=\theta_{0}$, find the optimal solution $\left(z_{0}, \lambda_{0}\right)$ by QP method.

Step 4: Define the active and inactive constraints in case of $\left(z_{0}, \lambda_{0}\right)$, and then build those matrices: $\tilde{G}, \tilde{W}, \tilde{S}$.

Step 5: Find $\left(\tilde{\lambda}, z^{*}\right)$ from (11) and (12).

Step 6: Characterize the $C R_{0}$ of $x$ from (13) in which the optimal solution is in (12).

Step 7: Redefine the current region be the $D-C R_{0}$ and go to step 2 .

Step 8: When all regions have been explored, exit.

\section{MAIN RESULTS}

\subsection{MPC controller for current sub-system} described by:

As mentioned in [17], current loop model of permanent magnet linear synchronous motor is

$$
\left\{\begin{array}{l}
\frac{d i_{s d}}{d t}=-\frac{R_{s}}{L_{s d}} i_{s d}+\left(\frac{2 \pi}{\tau} v\right) \frac{L_{s q}}{L_{s d}} i_{s q}+\frac{U_{s d}}{L_{s d}} \\
\frac{d i_{s q}}{d t}=-\frac{R_{s}}{L_{s q}} i_{s q}-\left(\frac{2 \pi}{\tau} v\right) \frac{L_{s d}}{L_{s q}} i_{s d}-\left(\frac{2 \pi}{\tau} v\right) \frac{\psi_{p}}{L_{s q}}+\frac{U_{s q}}{L_{s q}}
\end{array}\right.
$$


Denote that $i_{s d}^{d}, i_{s q}^{d}$ are desired outputs and $e_{s d}=i_{s d}-i_{s d}^{d}, e_{s q}=i_{s q}-i_{s q}^{d}$. Substituting into (30):

$$
\left\{\begin{array}{l}
\frac{d e_{s d}}{d t}=-\frac{R_{s}}{L_{s d}} e_{s d}+\left(\frac{2 \pi}{\tau} v\right) \frac{L_{s q}}{L_{s d}} e_{s q}+\frac{U_{s d}}{L_{s d}}-\left(-\frac{R_{s}}{L_{s d}} i_{s d}^{d}+\left(\frac{2 \pi}{\tau} v\right) \frac{L_{s q}}{L_{s d}} i_{s q}^{d}\right) \\
\frac{d e_{s q}}{d t}=-\frac{R_{s}}{L_{s q}} e_{s q}-\left(\frac{2 \pi}{\tau} v\right) \frac{L_{s d}}{L_{s q}} e_{s d}+\frac{U_{s q}}{L_{s q}}-\left(\frac{R_{s}}{L_{s q}} i_{s q}^{d}-\left(\frac{2 \pi}{\tau} v\right) \frac{L_{s d} i_{s d}^{d^{d}}+\psi_{p}}{L_{s q}}\right)
\end{array}\right.
$$

Define:

$$
A_{m}(t)=\left[\begin{array}{cc}
-\frac{R_{s}}{L_{s d}} & \left(\frac{2 \pi}{\tau} v(t)\right) \frac{L_{s q}}{L_{s d}} \\
-\frac{R_{s}}{L_{s q}} & -\left(\frac{2 \pi}{\tau} v(t)\right) \frac{L_{s d}}{L_{s q}}
\end{array}\right], B_{m}(t)=\left[\begin{array}{cc}
\frac{1}{L_{s d}} & 0 \\
0 & \frac{1}{L_{s q}}
\end{array}\right], u(t)=\left[\begin{array}{c}
\frac{U_{s d}}{L_{s d}}-\left(-\frac{R_{s}}{L_{s d} i^{d}}+\left(\frac{2 \pi}{\tau} v(t)\right) \frac{L_{s q}}{L_{s d} i^{d}{ }_{s q}}\right) \\
\frac{U_{s q}}{L_{s q}}-\left(\frac{R_{s}}{L_{s q}} i_{s q}^{d}-\left(\frac{2 \pi}{\tau} v(t)\right) \frac{L_{s d} d_{s d}{ }^{d} \psi_{p}}{L_{s q}}\right)
\end{array}\right]
$$

The current error model (31) is rewriten as:

$$
\frac{d x}{d t}=A_{m}(t) x(t)+B_{m} u(t)
$$

Obtaining discrete time model from (32) by using $\mathrm{ZOH}$ method:

$$
x(k+1)=\widehat{A}_{m}(k) x(k)+\widehat{B}_{m} u(k)
$$

Where: $\widehat{A}_{m}(k)=e^{A T_{s}}$ and $\widehat{B}_{m}=\int_{0}^{T_{s}} B_{m} d t=T_{s} B_{m}$

Then, we can apply MPC controller designed in 2.1 to current loop and the optimal control signal is presented in (20).

\subsection{Control design for outter loop}

Let us consider model of PMLSM proposed in [18]:

$$
\left\{\begin{array}{l}
\frac{d v}{d t}=\frac{p}{m}\left(F-F_{c}\right) \\
F=\frac{2 \pi}{\tau_{p}}\left[\psi_{p} i_{s q}+\left(L_{s d}-L_{s q}\right) i_{s d} i_{s q}\right] \\
\frac{d x}{d t}=v
\end{array}\right.
$$

Without loss of generality, we choose desired current in $d$-axis on $d-q$ coordinate: $i_{s d}^{d}=0$.

By letting $x_{1}=x-x_{d}, x_{2}=v-\dot{x}_{d}$ with $x_{d}$ is desired position, we obtain the model in state space as:

$$
\left\{\begin{array}{l}
\dot{x}_{1}=x_{2} \\
\dot{x}_{2}=a u+d-\ddot{x}_{d}
\end{array}\right.
$$

where $a=\frac{2 \pi}{\tau_{p}} \psi_{p}, u=i_{s q}$ and $d=-\frac{F_{c}}{m}$.

Lemma 1: As is presented in [7], by using controller (36) and disturbance observer (37), the state variables of system (35) converges to a ball centered at the origin. 


$$
\begin{aligned}
& u=\frac{1}{a}\left[-L_{1} \tanh \left(\mathrm{x}_{2}+L_{2} \tanh \left(\mathrm{x}_{1}\right)\right)-\hat{d}+\ddot{x}_{d}\right] \\
& \left\{\begin{array}{l}
\hat{d}(t)=\xi+K_{0} x_{2} \\
\dot{\xi}=-K_{0} \xi-K_{0}\left(a u-\ddot{x}_{d}+K_{0} x_{2}\right)
\end{array}\right.
\end{aligned}
$$

Proof:

Let $\bar{u}=a u-\ddot{x}_{d}$, we obtain:

$$
\left\{\begin{array}{l}
\dot{x}_{1}=x_{2} \\
\dot{x}_{2}=\bar{u}+\tilde{d}+\xi+K_{0} x_{2} \\
\dot{\xi}=-K_{0} \xi-K_{0}\left(\bar{u}-K_{0} x_{2}\right) \\
\hat{d}(t)=\xi+K_{0} x_{2}
\end{array}\right.
$$

where $\tilde{d}=d-\hat{d}$. Choose Lyapunov candidate function as follows:

$$
V=\frac{1}{2} L_{2}^{2} \tanh ^{2}\left(x_{1}\right)+\frac{1}{2}\left[x_{2}+L_{2} \tanh \left(x_{1}\right)\right]^{2}+\frac{1}{2} \tilde{d}^{2}
$$

Differentiating both side of (39) along solution of (38) with respect to $t$ :

$$
\begin{aligned}
\dot{V}= & -L_{2}^{3} \tanh ^{2}\left(x_{1}\right)\left(1-\tanh ^{2}\left(x_{1}\right)\right)+\left[x_{2}+L_{2} \tanh \left(x_{1}\right)\right](\bar{u}+d)+\tilde{d} \dot{\tilde{d}} \\
& +L_{2}\left(1-\tanh ^{2}\left(x_{1}\right)\right)\left[x_{2}+L_{2} \tanh \left(x_{1}\right)\right]^{2}
\end{aligned}
$$

Substituting (36) into (40):

$$
\begin{aligned}
& \dot{V} \leq-L_{2}^{3} \tanh ^{2}\left(x_{1}\right)\left(1-\tanh ^{2}\left(x_{1}\right)\right)-L_{1}\left[x_{2}+L_{2} \tanh \left(x_{1}\right)\right]^{2} \\
& +k_{1}\left[x_{2}+L_{2} \tanh \left(x_{1}\right)\right]^{2}+\frac{\tilde{d}^{2}}{4 k_{1}}+L_{2}\left[x_{2}+L_{2} \tanh \left(x_{1}\right)\right]^{2}+\tilde{d} \tilde{d}
\end{aligned}
$$

From (38):

$$
\dot{\hat{d}}=\dot{\xi}+K_{0} \dot{x}_{2}=-K_{0} \xi-K_{0}\left(\bar{u}+K_{0} x_{2}\right)+K_{0}\left(\bar{u}+\tilde{d}+\xi+K_{0} x_{2}\right)=K_{0} \tilde{d}
$$

Then, refer to:

$$
\dot{\tilde{d}}=\dot{d}-K_{0} \tilde{d}
$$

Rewriting (42) with the aid of (41):

$$
\dot{V} \leq-L_{2}^{3} \tanh ^{2}\left(x_{1}\right)\left(1-\tanh ^{2}\left(x_{1}\right)\right)+\left(-L_{1}+L_{2}+k_{1}\right)\left[x_{2}+L_{2} \tanh \left(x_{1}\right)\right]^{2}+\left(\frac{1}{4 k_{1}}-K_{0}\right) \tilde{d}^{2}+\tilde{d} \dot{d}
$$


And using the relation: $\tilde{d} \dot{d} \leq k_{2} \tilde{d}^{2}+\frac{\dot{d}^{2}}{4 k_{2}}$, we finally get:

$$
\dot{V} \leq-L_{2}^{3} \tanh ^{2}\left(x_{1}\right)\left(1-\tanh ^{2}\left(x_{1}\right)\right)+\left(-L_{1}+L_{2}+k_{1}\right)\left[x_{2}+L_{2} \tanh \left(x_{1}\right)\right]^{2}+\left(\frac{1}{4 k_{1}}-K_{0}+k_{2}\right) \tilde{d}^{2}+\frac{\dot{d}^{2}}{4 k_{2}}
$$

By selecting control parameter constant $L_{1}, L_{2}, K_{0}$ such that:

$$
L_{1}>L_{2}+k_{1}, K_{0}>k_{2}+\frac{1}{4 k_{1}}, k_{1}>0, k_{2}>0
$$

And assume that $|\dot{d}|<\infty$, since $k_{2}$ can be chosen arbitrarily large and from (59), Lemma 1 is proved.

\section{RESULTS AND ANALYSIS}

Base on the above conclusions, the simulation model of PMLSM and controller are constructed in Matlab environment. The parameters of PMLSM is given:

\begin{tabular}{ll}
\hline Parameter & Value \\
\hline Number of Pole & 2 \\
Pole step & $72 \mathrm{~mm}$ \\
Rotor mass & $3.5 \mathrm{~kg}$ \\
Phase coil Resistance & $3.1 \Omega$ \\
d-axis inductance & $4.1 \mathrm{mH}$ \\
q- axis inductance & $4.1 \mathrm{mH}$ \\
Flux & $0.8 \mathrm{~Wb}$ \\
\hline
\end{tabular}

Figure 1 and Figure 2 describe the responses of PMLSM in cases unconstrainted and constrainted MPC controller. In first case, Figure 1. display that actual trajectory's motor tracking designed trajectory very fast, but it requires the large started voltage. This is a reason to we must constraint the voltage input. Figure 2. shows responses of motor when the input voltage $u_{s q}$ is limited by $u_{s q \max }=90 \mathrm{~V}$.

Considering the desired trajectory of motors is expressed by: $x^{d}(t)=t$, we obtain following efficients:
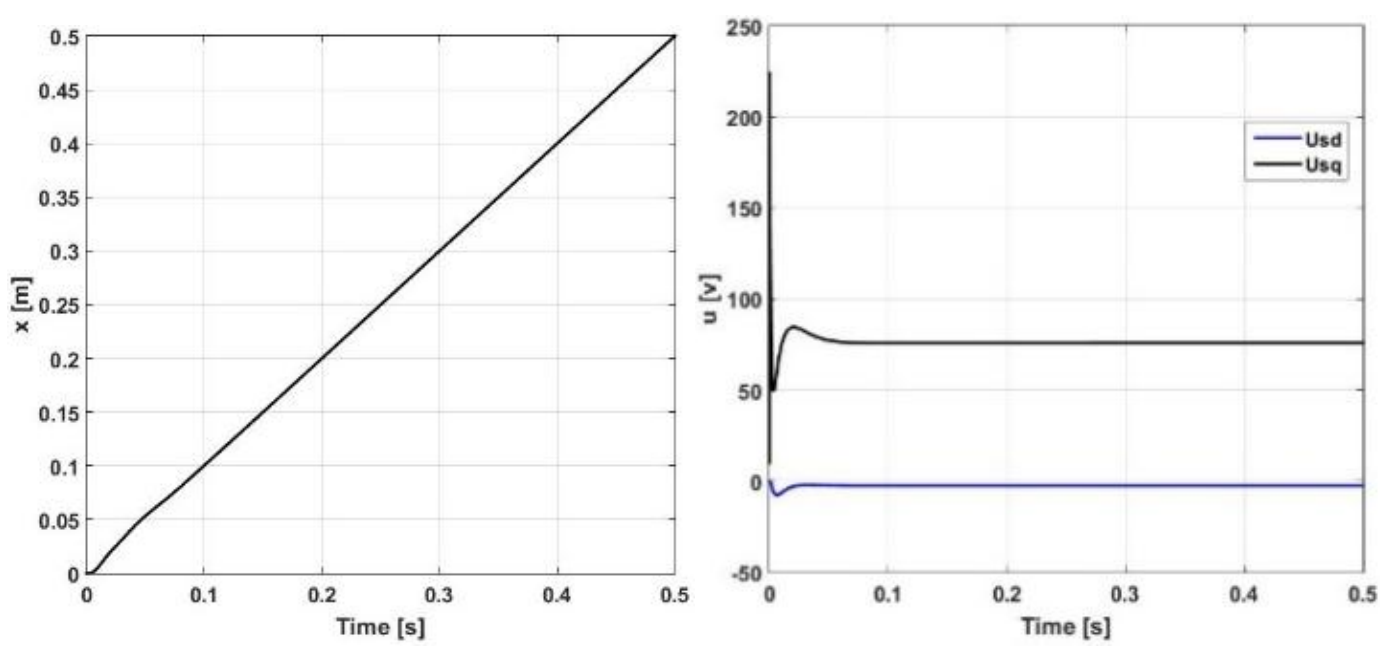

Figure 1. Actual trajectory and control signal with unconstrainted MPC 

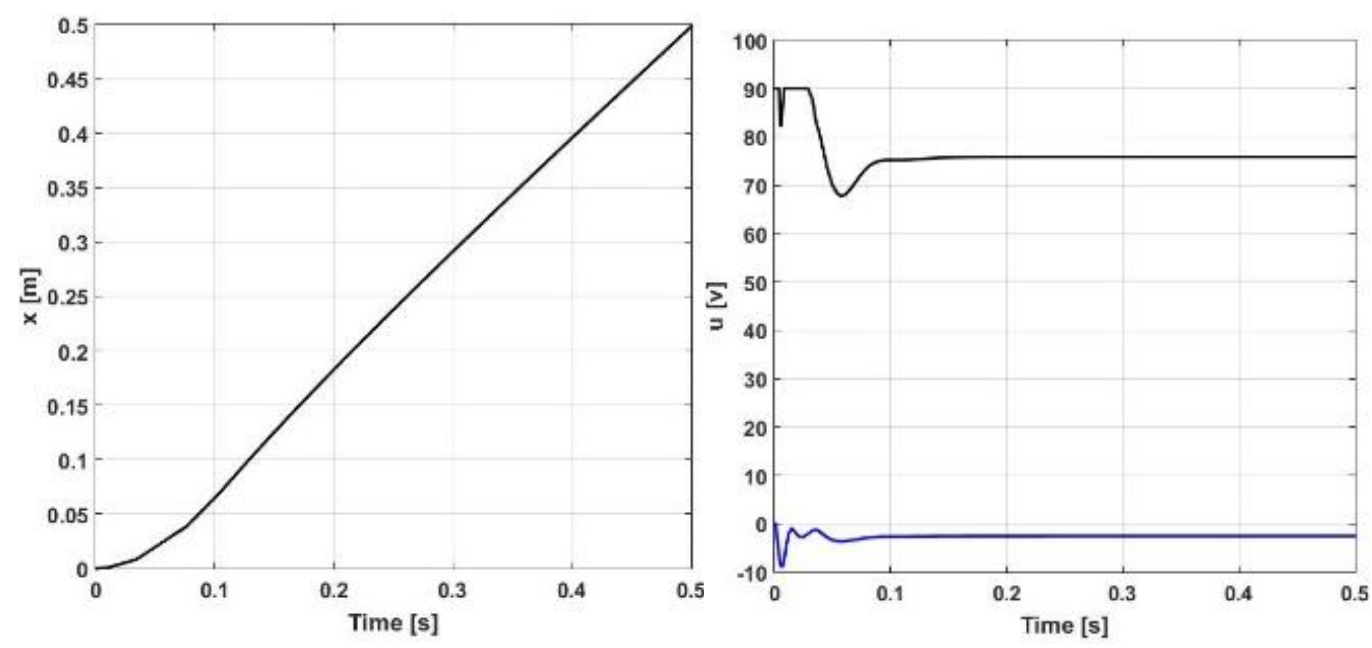

Figure 2. Actual trajectory and control signal with constrainted MPC

\section{CONCLUSION}

In this work, we apply cascade control strategy for polysolenoid linear motor to saparate the motor into outer and inner loop. The offline MPC controller based on MPP method was proposed for inner loop to make motor current to follow the reference signal from the outer controller. Optimization problem in the MPC controller was modified by using a Laguerre Model approach to reduce the number of optimal variables. The major advantage of our MPC controller lies in the ability to solve constraints problem and reducing amount of calculation because the optimal problem is offline solved. The outer controller was designed based on nonlinear damping method to guarantee the error between real and reference velocity converge to arbitrary small value.

\section{ACKNOWLEDGEMENTS} Technology.

This research was supported by Research Foundation funded by Thainguyen University of

\section{REFERENCES}

[1] Ng. Ph. Quang, J.-A. Dittrich; "Vector Control of Three-Phase AC Machines - System Development in the Practice," 2nd Edition 2015, Springer Berlin Heidelberg.

[2] Jacek F. Gieras, Zbigniew J. Piech, Bronislaw Tomczuk, "Linear Synchronous Motors Transportation and Automation Systems,"”2nd Edition. CRC press, 2011.

[3] I.Boldea; Linear Electric Machines, Drives, and MAGLEVs Handbook, CRC press, 2013.

[4] Daniel Ausderau, Polysolenoid - Linearantrieb mit genutetem Stator, Zurich. PhD Thessis, 2004.

[5] Huilai Li, Xiaomin Li, Zhiyuan Li, "Performance Assessment and Comparison of Two Types Linear Motors for Electromagnetic Catapult," TELKOMNIKA (Telecommunication, Computing, Electronics and Control), 2014 Apr., 12(4): 2506-2515

[6] Yang Zeqing, Liu Libing, Wangzuojie, Chen Yingshu, Xiao Quanyang, "Static and Dynamic Characteristic Simulation of Feed System Driven by Linear Motor in High Speed Computer Numerical Control Lathe," TELKOMNIKA (Telecommunication, Computing, Electronics and Control), 2013 July, 11(7): 3673-3683.

[7] Aymen Lachheb, Jalel Khediri, Lilia El Amraoui, "Performances Analysis of a Linear Motor for Sliding Door Application," International Journal of Power Electronics and Drive System (IJPEDS), 2017 Sep., 8(3): 1139-1146.

[8] Faa - Jeng Lin, Po - Hung Shen, "Adaptive fuzzy-neural-network control for a DSP-based permanent magnet linear synchronous motor servo drive," IEEE Transactions on Fuzzy Systems, 2006, pp. 481 - 495.

[9] Jul - Ki Seok, Jong - Kun Lee, Dong - Choon Lee, "Sensorless Speed Control of Nonsalient Permanent Magnet Synchronous Motor Using Rotor - Position - Tracking PI Controller," IEEE Transactions on Industrial Electronics, Vol. 53, No. 2, pp.399 - 405, 2006.

[10] Yuan - Rui Chen, Jie Wu, Nobert Cheung (2004), "Lyapunov's Stability Theory - Based Model Reference Adaptive Control for Permanent Magnet Linear Motor Drives," Proc of Power Electronics Systems and Application, 2004, pp. $260-266$ 
[11] Chin - I Huang, Li - Chen Fu, "Adaptive Back stepping Speed/Position Control with Friction Compensation for Linear Induction Motor," in Proceeding of the $41^{\text {st }}$ IEEE Conference on Decision and Control, 2002, USA, pp. 474 -479 .

[12] Gerardo Tapia, Arantxa Tapia (2007), "Sliding - Mode Control for Linear Permanent - Magnet motor Position Tracking," Proc of the IFAC World Congress.

[13] Liuping Wang; "Model Predictive Control System Design and Implementation Using MATLAB," Springer-Verlag London Limited, 2009.

[14] Hossein Komijani, Saeed Masoumi Kazraji, Ehsan Baneshi, Milad Janghorban Lariche, "Modeling and State Feedback Controller Design of Tubular Linear Permanent Magnet Synchronous Motor", International Journal of Power Electronics and Drive System (IJPEDS), 2016 Dec., 7(4): 1410-1419.

[15] Alexandra Grancharova, Arne Hohansen, Explicit "Nonlinear Model Predictive Control - Theory and Applications". Springer, 2012, ch. 1.

[16] H. K. Khalil. Nonlinear Systems. 3rd ed. Upper Saddle River, NJ:Prentice-Hall, 2002

[17] Quang H. Nguyen, Nam P. Dao, Ty T. Nguyen, Hung M. Nguyen, Hien N. Nguyen, Tan D. Vu, "Flatness Based Control Structure for Polysolenoid Permanent Stimulation Linear Motors," SSRG International Journal of Electrical and Electronics Engineering, Volume-3 Issue-12, 2016, pp 31-37.

[18] Quang H. Nguyen, Nam P. Dao, Hung M. Nguyen, Hien N. Nguyen, Ty T. Nguyen, Chi P. Nguyen, "Design an Exact Linearization Controller for Permanent Stimulation Synchronous Linear Motor Polysolenoid," SSRG International Journal of Electrical and Electronics Engineering, Volume-4 Issue-1, 2017, pp. 7-12.

\section{BIOGRAPHIES OF AUTHORS}
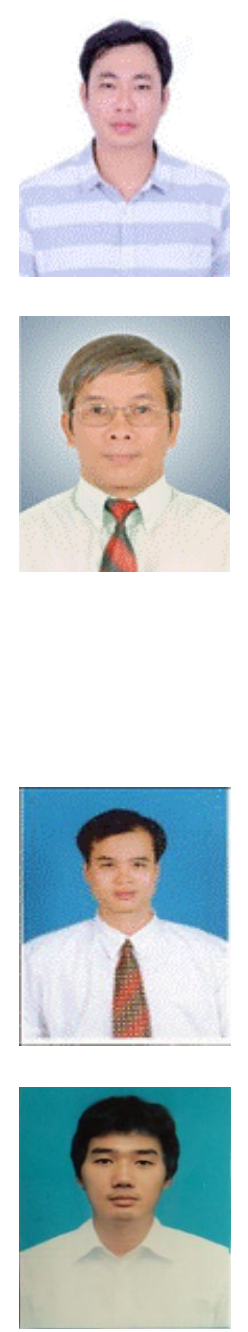

Nguyen Hong Quang received the B.S degree in electrical engineering from Thai Nguyen University of technology (TNUT), Vietnam, in 2007, the Master's degree in control engineering and automation from Hanoi University of Science and Technology (HUST), Viet Nam, in 2012. He is currently with TNUT as Ph.D student and Lecturer. His Research Interests are Electrical Drive Systems, Adaptive Dynamic Programming Control, Robust Nonlinear Model Predictive Control.

Nguyen Phung Quang received his Dipl.-Ing. (Uni.), Dr.-Ing. and Dr.-Ing. habil. degrees from TU Dresden, Germany in 1975, 1991 and 1994 respectively. Prior to his return to Vietnam, he had worked in Germany industry for many years, contributed to create inverters REFU 402 Vectovar, RD500 (REFU Elektronik); Simovert 6SE42, Master Drive MC (Siemens). From 1996 to 1998, he served as lecturer of TU Dresden where he was conferred as Privatdozent in 1997. He joined Hanoi University of Science and Technology in 1999, as lecturer up to now. He is currently a professor of HUST and honorary professor of TU Dresden. He was author/co-author of more than 170 journal and conference papers; 8 books with three among them was written in German and one in English entitled "Vector Control of Three-Phase AC Machines - System Development in the Practice" published by Springer in 2008, and 2nd edition in June 2015. . His Research Interests are Electrical Drive Systems, Motion Control, Robotic Control, Vector Control of Electrical Machines, Wind and Solar Power Systems, Digital Control Systems, Modeling and Simulation.

Dao Phuong Nam obtained Doctor degree on January - 2013 at Hanoi University of Science and Technology (Vietnam). Currently, he holds the position as lecturer at Hanoi University of Science and Technology, Vietnam. His research interests include control of robotic systems and robust/adaptive, optimal control $\mathrm{He}$ is author/co-author of more than 70 papers (Journals, Conferences,...)

Nguyen Thanh Binh received the B.S and M.S degree in control engineering and automation from Hanoi University of Science and Technology (HUST), Viet Nam, in 2014 and 2017. Currently, he holds the position as lecturer at Thuyloi University, Vietnam. Since 2018, He is currently with University of Ulsan, Ulsan, Korea as PhD student. His research interests include Control of land, air, underwater vehicles, and Robust Nonlinear Model Predictive Control. 